\title{
Are we approaching a new era in the treatment of anemia of chronic kidney disease patients?
}

\author{
Francesco Locatelli, Lucia Del Vecchio
}

Alessandro Manzoni Hospital, ASST Lecco, Lecco, Italy

Correspondence to: Prof. Dr. Francesco Locatelli, MD. Alessandro Manzoni Hospital, ASST Lecco, Via dell'Eremo 9, 23900 Lecco, Italy.

Email: f.locatelli@asst-lecco.it.

Provenance: This is an invited article commissioned by the Section Editor Dr. Linpei Jia (Department of Nephrology, Xuanwu Hospital of Capital Medical University, Beijing, China).

Comment on: Chen N, Hao C, Peng X, et al. Roxadustat for Anemia in Patients with Kidney Disease Not Receiving Dialysis. N Engl J Med 2019;381:1001-10.

Submitted Sep 09, 2019. Accepted for publication Sep 20, 2019.

doi: 10.21037/atm.2019.09.119

View this article at: http://dx.doi.org/10.21037/atm.2019.09.119

In the middle of XX century, the clinical implementation of dialysis arrived as the astonishing first possibility to allow the patients with end-stage chronic kidney disease (CKD) to survive despite their failing vital organs: the kidneys.

Soon it was evident that while dialysis could roughly mimic the depurative capacity of the kidneys, it was unable to replace its endocrine activity. In those days, the lack of production of erythropoietin (EPO) was the most important clinical problem of advanced CKD. The patients were able to survive thanks to dialysis, however, their quality of life was poor, not only because of the need of dialysis and the associated complications, but also because of symptoms.

Compared to nowadays, the depurative capacity of dialysis was inadequate and the biocompatibility of the system limited: inflammation and uremic toxins further aggravated anemia by causing the shortening of red cell survival (especially in dialysis patients) and bone marrow chronic depression. Subclinical gastro-intestinal bleeding, blood losses during the dialysis session, surgical interventions for vascular access or peritoneal catheter, blood draws, hyperparathyroidism, iron and vitamin deficiencies all also contributed to the severity of anemia.

In those days, a large majority of the patients were transfusion-dependent and the hemoglobin $(\mathrm{Hb})$ levels were below $7 \mathrm{~g} / \mathrm{dL}$ in a large proportion of cases. Hemosiderosis, which was due to transfusion-related iron accumulation in the liver and heart, was a frequent complication, together with chronic viral hepatitis.

\section{Erythropoiesis-stimulating agents (ESAs)}

The treatment of anemia in CKD had greatly advanced by the introduction of ESAs. These agents have dramatically reduced the need for transfusions and their related side effects, including viral infections and allo-antibody production, and improved quality of life, reducing many of the symptoms previously attributed to uremic intoxication.

While the availability of recombinant human erythropoietin is considered the most important achievement in the treatment of patients with end stage CKD, a part dialysis and transplantation, these agents also have drawbacks due to a possible increase in the risk of cardiovascular events, thrombosis and, although occasionally, cancer (1). The risk is higher when $\mathrm{Hb}$ normalization was set as a target and/ or high doses were used (1). In this respect, there is still a significant percentage of CKD patients who are hyporesponsive to ESAs, mainly because of inflammation, causing functional iron deficiency (2).

Pure red cell aplasia is also a rare and feared complication of ESAs; its prevention can be obtained with strict and accurate manufacturing processes and careful cold chain (3).

Finally, ESA treatment is still expensive.

The first generation of short-acting ESA (epoetin alfa and beta) were introduced into the market at the end of the eighties. Afterwards, two second-generation ESAs with longer half-life were developed: the hyperglycosylated version of epoetin alfa (darbepoetin alfa) and methoxypolyethylene glycol-epoetin beta. They can be administered 
on a less frequent basis, up to once a month. All ESAs increase red cell production by stimulating the EPO receptor. However, they have different pharmacokinetic and pharmaco-dynamic characteristics, translating into different half-life and different receptor affinity, resulting in different ESA blood concentration levels following IV or SC administration $(4,5)$ and possibly different clinical or subclinical effects besides anemia correction.

Until recently, the possibility that the ESA molecules could have a different effects and safety profile have been little investigated (6-8). The MIRCERA PASS is a very recently published large, randomized, non-inferiority trial aimed at comparing all-cause mortality and cardiovascular risk of methoxy polyethylene glycol-epoetin beta in respect to other ESAs (8). Its design was mandated by the Food and Drug Administration and by the European Medicines Agency to verify post-approval safety. The study enrolled 2818 CKD dialysis and non-dialysis (ND) patients, who were randomized to either methoxy polyethylene glycolepoetin beta or to a reference agent and followed for a median of 3.4 years (maximum, 8.4 years). Once-monthly methoxy polyethylene glycol-epoetin beta was found noninferior to the other ESAs on the rates of major adverse cardiovascular events or all-cause mortality, challenging the results of a large observational study on the Japanese Registry database, suggesting higher mortality associated with long acting ESAs use.

\section{Severe anemia is still a factor of concern}

Left untreated, anemia is associated with poor prognosis (9-12). Recently, Chen et al. (13), in reporting the data of a phase-III clinical trial conducted at 29 sites in China, underlined the relevance of a paper (13), underlying an inadequate anemia treatment in China. Indeed, it was matter of concern the very low mean $\mathrm{Hb}$ levels of the patients who start dialysis in China $(7.3 \mathrm{~g} / \mathrm{dL}$ in Guangzhou, $8.2 \mathrm{~g} / \mathrm{dL}$ in Beijing, and $7.7 \mathrm{~g} / \mathrm{dL}$ in Shanghai). The situation is even worse in rural China, where the patients start dialysis with a mean $\mathrm{Hb}$ value of only $5.9 \mathrm{~g} / \mathrm{dL}$; these patients die or develop heart failure at a double rate than those living in urban areas who have higher $\mathrm{Hb}$ levels (a mean of $8.4 \mathrm{~g} / \mathrm{dL}$ ) (14).

This information is of paramount importance, considering that, in the study by Chen et al. (13), the definition of anemia was very conservative (Hb levels $<10 \mathrm{~g} / \mathrm{dL}$ ); however, mean $\mathrm{Hb}$ values at baseline were well below this limit and likely the majority of the patients were already experiencing symptoms related to anemia. This inclusion criterion was possibly chosen taking into consideration the $\mathrm{Hb}$ level below which KDIGO Guidelines recommend deciding whether to start or not ESA therapy on the basis of the rate of fall of $\mathrm{Hb}$ concentration, prior response to iron therapy, transfusion needs, ESA risks and the presence of symptoms attributable to anemia.

This KDIGO recommendation is not universally accepted; the lack of a lower Hb limit at which ESA treatment should be better started may expose patients to the negative effects of severe anemia. Other bodies such as the European Renal Best Practice (ERBP) (15) and the National Institute for Health and Care Excellence (NICE) (16) suggest a $\mathrm{Hb}$ target range of $10-12 \mathrm{~g} / \mathrm{dL}$ and to start ESA therapy when $\mathrm{Hb}$ is below $10 \mathrm{~g} / \mathrm{dL}$.

\section{The burden of the cost of treatment}

ESA treatment is expensive. This is partially due to the fact that epoetins are biological drugs with a complex and delicate manufacturing process involving the recombinant DNA technique. Moreover, their distribution and stocking need the implementation and maintaining of a strict cold chain. The burden of the cost is of paramount importance, as testified by the introduction and penetrance around the world of the biosimilars, with the only advantage of reducing the cost in respect to their originators.

The introduction of biosimilars into the market has had the advantage of giving a cheaper option and contributed to reduce the cost of the originators as well. However, biosimilars are necessarily produced by the same manufacturing process of their originators; consequently they remain biological expensive drugs. Moreover, in many Western countries their penetrance into the market has been less than the expected. In poorer countries, where health system rules are less strict, several biosimilars (better defined as copies) are produced and administered, although their safety is questionable (17). Even so, just a minority of the patients are given ESA treatment or they receive it at little doses unable to achieve adequate anemia correction. In these countries, anemia treatment is unfortunately not so different from that the patients received in the seventies or eighties.

In this respect, the very low $\mathrm{Hb}$ levels observed at the start of dialysis in China are not only due to the concern of Chinese nephrologists about the safety around ESA use, but more likely to the unaffordable economic impact of treating an impressively large CKD population, (around 120 million 
people, many of the suffering of anemia).

\section{Prolyl hydroxylase inhibitors: (PHDi) oral agents activating the hypoxia inducible factor}

A better understanding of the response of the body to hypoxia and the related oxygen sensing system has clarified the central role of the hypoxia-inducible factors (HIF) in EPO production. PHDi produce effects in the body that are similar to those occurring at high altitude exposure, inducing the bone marrow to produce more red blood cells. The HIF system not only upregulates EPO receptors, but also increases iron mobilization from the gut and macrophages through hepcidin-dependent and independent pathways (18). Hepcidin is usually upregulated in CKD and limits iron absorption and mobilization contributing to functional iron deficiency; PHDi have been shown to significantly reduce hepcidin levels, likely through indirect mechanisms (18).

\section{Roxadustat}

Several PHDi are currently under advanced development phase; among them, roxadustat is the most advanced one, with some phase III studies already concluded. It is approved for clinical use in China for dialysis and ND patients.

The paper by Chen et al. (13) is a relevant step in increasing the knowledge on the clinical use of rodaxustat in the Chinese population. One hundred fifty-four patients with CKD were assigned to either roxadustat or placebo (2:1 ratio) for 8 weeks. This phase-III study showed an increase of $1.9 \pm 1.2 \mathrm{~g} / \mathrm{dL}$ in mean $\mathrm{Hb}$ values from baseline in the group randomized to roxadustat compared to a mean decrease of $0.4 \pm 0.8 \mathrm{~g} / \mathrm{dL}$ in the placebo group. The mean reduction from baseline in the hepcidin level was $56.14 \pm 63.40 \mathrm{ng} / \mathrm{mL}$ in the roxadustat group and $15.10 \pm 48.06 \mathrm{ng} / \mathrm{mL}$ in the placebo group. Hyperkalemia and metabolic acidosis occurred more frequently in the roxadustat group than in the placebo group; the reasons for this are unknown. Hb levels were then effectively maintained during the 18 -week open-label period. Similar findings were reported for the dialysis population in comparison to epoetin alfa (19).

Differing from ESAS, which stimulate the EPO receptors with over-physiological EPO levels, PHDi stimulate endogenous EPO production, exposing patients to lower plasma levels, while also regulating iron metabolism.
This peculiarity, together with other possible mechanisms, leave open the possibility that roxadustat cardiovascular profile may be potentially better in comparison to ESA. In the study by Chen et al. (13) roxadustat does not have hypertensive effects in comparisons to placebo and reduces serum cholesterol levels. In May 2019, a press release on the preliminary data of a pooled analyses of the global phase III program (over 4,300 NDD and around 4,000 DD patients) showed no clinically meaningful difference in MACE between roxadustat and placebo (20). The publication of the full data set is awaited by the end of the year.

Compliance is a major aspect to be considered in evaluating the clinical impact of a new drug. The benefit of the oral administration, which avoids subcutaneous injections in ND patients and eliminates the cold chain, should be balanced against the fact that CKD patients usually take many oral drugs, including anti-hypertensive, cholesterol lowering drugs and iron.

Despite these encouraging findings, PHDi are still surrounded by some uncertainties, partly because the HIF pathway is complex and not fully explored and understood. The potential stimulation of the Vascular Endothelium Grow Factor (VEGF) could be a matter of concern, particularly considering a possible acceleration of diabetic retinopathy and cancer development. However, the available data are reassuring, with relatively low VEGF plasma levels and no worsening of diabetic retinopathy till now.

\section{Future avenues of PHDi}

PHDi, including roxadustat, seems to have the capability of correcting anemia in CKD inflamed patients; this is an unmet need in the treatment of anemia in CKD patients. This characteristic could open the door to the treatment of other types of anemia, including anemia of the aging population, heart failure and of chronic diseases more in general. This potential large population to be treated could be the explanation why so many companies are developing PHDi, while the use of ESAs has dramatically decreased, especially in non-dialysis patients.

\section{Acknowledgments}

None.

\section{Footnote}

Conflicts of Interest: Prof. Locatelli is Member of an advisory 
Board of Amgen, GSK, Roche, Vifor Pharma and invited Speaker at meeting supported by Amgen, Astellas-AstraZeneca, GSK, Mitsubishi, Roche, Vifor pharma. Dr. Lucia Del Vecchio had been member of Advisory Boards for DOC, Roche, Astellas and invited speaker at meetings supported by DOC, Roche, Astellas, Vifor Pharma. She is national leader for the ASCEND-ND study supported by JSK.

Ethical Statement: The authors are accountable for all aspects of the work in ensuring that questions related to the accuracy or integrity of any part of the work are appropriately investigated and resolved.

\section{References}

1. Del Vecchio L, Locatelli F. An overview on safety issues related to erythropoiesis-stimulating agents for the treatment of anaemia in patients with chronic kidney disease. Expert Opin Drug Saf 2016;15:1021-30.

2. Parfrey PS. Erythropoietin-stimulating agents in chronic kidney disease: a response to hyporesponsiveness. Semin Dial 2011;24:495-97.

3. Pollock C, Johnson DW, Hörl WH, et al. Pure red cell aplasia induced by erythropoiesis-stimulating agents. Clin J Am Soc Nephrol 2008;3:193-9.

4. Besarab A, Reyes CM, Hornberger J. Meta-analysis of subcutaneous versus intravenous epoetin in maintenance treatment of anemia in hemodialysis patients. Am J Kidney Dis 2002;40:439-46.

5. Locatelli F, Del Vecchio L, Marai P. Clinical experience with darbepoetin-alfa (Aranesp). Contrib Nephrol 2002;(137):403-7.

6. Wilhelm-Leen ER, Winkelmayer WC. Mortality risk of darbepoetin alfa versus epoetin alfa in patients with CKD: systematic review and meta-analysis. Am J Kidney Dis 2015;66:69-74.

7. Saglimbene VM, Palmer SC, Ruospo M, et al. Continuous erythropoiesis receptor activator (CERA) for the anaemia of chronic kidney disease. Cochrane Database Syst Rev 2017;8:CD009904.

8. Locatelli F, Hannedouche T, Fishbane S, et al. Cardiovascular safety and all-cause mortality of methoxy polyethylene glycol-epoetin beta and other erythropoiesisstimulating agents in anemia of CKD: a randomized noninferiority trial. Clin J Am Soc Nephrol 2019. [Epub ahead of print].

9. Foley RN, Parfrey PS, Harnett JD, et al. The impact of anemia on cardiomyopathy, morbidity, and mortality in end-stage renal disease. Am J Kidney Dis 1996;28:53-61.

10. Locatelli F, Pisoni RL, Combe C, et al. Anaemia in haemodialysis patients of five European countries: association with morbidity and mortality in the Dialysis Outcomes and Practice Patterns Study (DOPPS). Nephrol Dial Transplant 2004;19:121-32.

11. Walker AM, Schneider G, Yeaw J, et al. Anemia as a predictor of cardiovascular events in patients with elevated serum creatinine. J Am Soc Nephrol 2006;17:2293-98.

12. Levin A, Thompson CR, Ethier J, et al. Left ventricular mass index increase in early renal disease: impact of decline in hemoglobin. Am J Kidney Dis 1999;34:125-34.

13. Chen N, Hao C, Peng X, et al. Roxadustat for anemia in patients with kidney disease not receiving dialysis. N Engl J Med 2019;381:1001-10.

14. Zhang W, Gong Z, Peng X, et al. Clinical characteristics and outcomes of rural patients with ESRD in Guangxi, China: one dialysis center experience. Int Urol Nephrol 2010;42:195-204.

15. Locatelli F, Bárány P, Covic A, et al.; ERA-EDTA ERBP Advisory Board. Kidney Disease: Improving Global Outcomes guidelines on anaemia management in chronic kidney disease: a European Renal Best Practice position statement. Nephrol Dial Transplant 2013;28:1346-59.

16. Available online: https://www.nice.org.uk/guidance/ng8 last accessed on the 4th of September, 2019.

17. Praditpornsilpa K, Tiranathanagul K, Kupatawintu P, et al. Biosimilar recombinant human erythropoietin induces the production of neutralizing antibodies. Kidney Int 2011;80:88-92.

18. Haase VH. HIF-prolyl hydroxylases as therapeutic targets in erythropoiesis and iron metabolism. Hemodial Int 2017;21 Suppl 1:S110-24.

19. Chen N, Hao C, Liu BC, et al. Roxadustat treatment for anemia in patients undergoing long-term dialysis. $\mathrm{N}$ Engl J Med 2019;381:1011-22.

20. Available online: https://pharmaphorum.com/news/ astrazeneca-shows-cardiovascular-safety-for-roxadustat/. Last accessed on the 4th of September, 2019.

Cite this article as: Locatelli F, Del Vecchio L. Are we approaching a new era in the treatment of anemia of chronic kidney disease patients? Ann Transl Med 2019;7(Suppl 8):S333. doi: 10.21037/atm.2019.09.119 